\title{
In vitro evaluation of natural and methylated cyclodextrins as buccal permeation enhancing system for omeprazole delivery
}

\author{
Ana Figueiras ${ }^{a}$, Juliane Hombach ${ }^{b}$, Francisco Veiga ${ }^{\mathrm{a}, *}$, Andreas Bernkop-Schnürch ${ }^{\mathrm{b}}$ \\ a Department of Pharmaceutical Technology, University of Coimbra, Coimbra, Portugal \\ ${ }^{\mathrm{b}}$ Institute of Pharmacy, University of Innsbruck, Austria
}

\section{A R T I C L E I N F O}

\section{Article history:}

Received 26 March 2008

Accepted in revised form 7 August 2008

Available online 29 August 2008

\section{Keywords:}

Buccal mucosa

Cyclodextrins

L-Arginine

Omeprazole

Permeation

Stability

\begin{abstract}
A B S T R A C T
In this work the enhancing effect of cyclodextrins on the buccal permeation of a hydrophobic model drug, omeprazole was studied. First, the influence of the complexation with cyclodextrins in the absence and in the presence of an alkali agent, L-arginine, on the drug stability was checked at neutral conditions since omeprazole alone is only stable in basic conditions. In vitro transbuccal permeation of omeprazole non-complexed and complexed with $\beta$ - and methyl- $\beta$-cyclodextrin and in presence of L-arginine was examined using freshly obtained porcine buccal mucosa. Tissue viability after incubation with sample solutions was assessed using a MTT (3-[4,5-dimethylthiazol-2-yl]-2,5-diphenyltetrazolium bromide) biochemical assay and histological evaluation. The toxicity of the sample solutions on buccal mucosa was evaluated by measuring lactate dehydrogenase activity. The present results show that complexation with cyclodextrins increases drug stability at neutral conditions; furthermore, L-arginine contributed to higher drug stability. Permeation studies indicate an increase on drug permeation in complexed form of 1.1- and 1.7 -fold for $\beta$-cyclodextrin and methyl- $\beta$-cyclodextrin, respectively. The presence of $\mathrm{L}$-arginine increases drug permeation 1.4-fold in omeprazole complexed with $\beta$-cyclodextrin and 2.4-fold in the inclusion complex formed with methyl- $\beta$-cyclodextrin. The cell viability of the buccal mucosa after a $3 \mathrm{~h}$ incubation period, with all sample solutions, remained around $70 \%$ and lactate dehydrogenase assay showed that studied cyclodextrins, even in the presence of an alkali agent are not cytotoxic for porcine buccal mucosa. Histological evaluation of the tissue demonstrated that the buccal epithelium remains viable after $3 \mathrm{~h}$ of incubation with sample solutions.
\end{abstract}

(c) 2008 Elsevier B.V. All rights reserved.

\section{Introduction}

Buccal mucosa is a potential site for drug absorption in alternative to oral drug delivery. Active molecules administered through the buccal mucosa pass directly into the systemic circulation, thereby minimizing the first hepatic pass and adverse gastro-intestinal effects [1,2]. Other important advantages are the low enzymatic activity, suitability for drugs or excipients that mildly and reversibly damage or irritate the mucosa, painless administration, easy drug withdrawal, facility to include permeation enhancer/enzyme inhibitor or $\mathrm{pH}$ modifier in the formulation and versatility in designing as multidirectional or unidirectional release systems for local or systemic actions [3]. However, lower permeability of the buccal mucosa to large molecules can be problematic in order to achieve therapeutic levels of such molecules. Buccal permeation can be increased by using various penetration enhancers. Recently,

\footnotetext{
* Corresponding author. Department of Pharmaceutical Technology, Faculty of Pharmacy, University of Coimbra, 3000-295 Coimbra, Portugal. Tel.: +351 239855085; fax: +351239855099.

E-mail address: fveiga@ci.uc.pt (F. Veiga).
}

cyclodextrins have been classified as a new class of penetration enhancers [4-6]. These molecules are cyclic oligosaccharides with a hydrophilic outer surface and a hydrophobic central cavity. The hydrophilic exterior of the cyclodextrin molecules makes them water-soluble while the hydrophobic cavity provides a microenvironment for appropriate sized non-polar molecules [7]. Cyclodextrins are able to form dynamic molecular inclusion complexes with many drugs by incorporating the drug molecule, or commonly a lipophilic moiety of the molecule, into the central cavity [8]. These non-covalent complexes offer a variety of physicochemical advantages over the unmodified drugs such as the possibility to increase their water solubility and stability [9]. It is generally recognized that cyclodextrins act as true carriers by keeping the hydrophobic drug molecules in solution and deliver them to the surface of the biological membrane, where they partition into the membrane [10]. Cyclodextrins can enhance drug permeation by increasing drug availability and stability at the surface of the biological barriers [11]. However, derivative cyclodextrins, especially methylated cyclodextrins, act as absorption enhancers by different pathways. These hydrophobic cyclodextrins act as absorption enhancers, probably, by transiently changing membrane perme- 
ability, overcoming the aqueous diffusion barrier and opening tight junctions [12]. During cell differentiation process in buccal mucosa, small organelles called membrane coating granules (MCGs) composed of non-lamellar lipid sacks are formed in intercellular spaces of the non-keratinized regions [13,14]. These MCGs, first observed in epidermis, are not different from those observed in the skin and are believed to provide an intercellular permeability barrier to buccal mucosa [15]. Methyl- $\beta$-cyclodextrin, a more hydrophobic cyclodextrin, can permeate the buccal mucosa and to form inclusion complexes with hydrophobic molecules, namely lipids from the cellular membrane, interacting strongly with these lipids; it could modify buccal mucosa permeability and could act as penetration enhancer for the buccal route.

Omeprazole (OME) a substituted benzimidazole, has been shown to effectively suppress gastric acid secretion by inhibiting the $\mathrm{H}^{+} \mathrm{K}^{+}$-ATPase (proton pump), in the parietal cells $[16,17]$. The bioavailability of OME following oral administration is usually very low, since it degrades quickly in the acidic environment of the stomach and undergoes hepatic first-pass metabolism. As an attempt to improve the oral bioavailability of OME various oral formulations have been developed over the years. However, these oral formulations revealed to have a large intra-individual variation in drug plasma concentration in human subjects $[18,19]$.

The aim of this study is to evaluate the potential enhancement effect of the cyclodextrins on the buccal mucosa permeation of OME. Inclusion complexes between OME and both cyclodextrins in absence and in presence of an alkali agent, L-arginine (ARG), were prepared by a freeze-drying method [20]. The effect of $\beta$-cyclodextrin $(\beta C D)$ and methyl- $\beta$-cyclodextrin $(M \beta C D)$ in the absence and in the presence of ARG on the OME stability was assessed at neutral conditions by high performance liquid chromatography (HPLC). Permeation studies were performed using two different cyclodextrins, a natural cyclodextrin, $\beta C D$ and a methylated derivative, $M \beta C D$, in order to compare their permeation enhancing properties. Viability, integrity and cytotoxicity studies were carried out to investigate possible morphologic changes of the buccal mucosa, after contact with the complexed OME in the absence and presence of ARG. Structural alterations in the buccal mucosa were also considered and supported by histological studies.

\section{Materials and methods}

\subsection{Materials}

$\beta C D$ (Beta-cyclodextrin, KLEPTOSE ${ }^{\circledR}, M_{\mathrm{w}}=1135$ ) and $\mathrm{M} \beta \mathrm{CD}$ (Methyl- $\beta$-cyclodextrin, CRYSMEB ${ }^{\circledR}, M_{\mathrm{w}} \sim 1190$, with an average degree of substitution of 0.5 ) were kindly donated by Roquette (Lestrem, France) and Omeprazole (OME, $M_{\mathrm{w}}=345.42$ ) was kindly donated by Belmac Laboratory, S.A. (Madrid, Spain). L-Arginine (ARG) was purchased from Panreac (Santiago de Compostela, Spain). Thiazolyl blue tetrazolium bromide (MTT), fluorescein isothiocyanate-dextran 40 (FD40, average $M_{\mathrm{w}}$ 40.000) were purchased from Sigma (St. Louis, MO, USA). The cytotoxicity detection kit (Dye solution + Catalyst) was purchased from Roche. All other reagents (chemicals and solvents) were of analytical grade.

\subsection{Preparation of inclusion complexes}

Solid inclusion complexes were prepared by freeze-drying method. Systems were prepared in a stoichiometry 1:1 (drug:cyclodextrin) according to previous phase solubility studies [21] and ARG was added in a molar proportion 6:1, relatively to OME. The same systems were prepared in the absence of ARG to observe the influence of the alkali agent on the drug stability and permeation. All the clear solutions were frozen by immersion in an ethanol-bath at $-50{ }^{\circ} \mathrm{C}$ (Shell Freezer, Labconco, Freezone ${ }^{\circledR}$ model 79490) and the frozen solutions were lyophilized in a freeze-dryer (Lyph-lock 6 apparatus, Labconco) for $72 \mathrm{~h}$.

\subsection{Stability studies}

The stability of OME alone was checked at three different $\mathrm{pH}$ values: 7.0, 7.9 and 9.0. After, stability of OME alone, complexed with $\beta C D$ or $M \beta C D$ in absence and in presence of ARG was examined at $37{ }^{\circ} \mathrm{C}$ in $40 \mathrm{mM}$ of Bis-Tris buffer (bis[2-hydroxyethyl]iminotris[hydroxymethyl]methane) at $\mathrm{pH} 7.0 \pm 0.5$. Solutions containing $100 \mu \mathrm{g} / \mathrm{mL}$ of OME in free and complexed state were prepared. These solutions were stirred at $300 \mathrm{rpm}$ during $8 \mathrm{~h}$ at $37^{\circ} \mathrm{C}$. Samples were collected every hour and analyzed by HPLC.

\subsection{High performance liquid chromatography (HPLC)}

The official HPLC method described in the USP XXX [22] was used for quantification of the drug. An Elite Lachrom Liquid Chromatograph (Merck, Hitachi) system consisting of a quaternary pump (L2130), with a L2450 programmable multiple wavelength detector set at $300 \mathrm{~nm}$ and an autosampler L2200 was used. The separation was carried out at room temperature and the column used was a reverse-phase Purospher ${ }^{\circledR}$ RP-18 endcapped $(5 \mu \mathrm{m})$, $125 \mathrm{~cm} \times 4 \mathrm{~mm}$. The mobile phase was a mixture of phosphate buffer $(\mathrm{pH} 7.6 \pm 0.5)$ and acetonitrile $(75: 25, \mathrm{v} / \mathrm{v})$, filtered through $0.20 \mu \mathrm{m}$ nylon filters, degassed and pumped at a constant flow rate of $1 \mathrm{~mL} \mathrm{~min}{ }^{-1}$. The chromatograms were recorded and the peak area response was measured using an automatic integrator. The injection volume was $20 \mu \mathrm{l}$ for all standards and samples.

\subsubsection{Validation of the HPLC method}

The HPLC method was validated by using the following analytical parameters: linearity, precision, accuracy, repeatability and specificity. Linearity was evaluated by calculation of a regression line using the least squares method. Calibration curve was obtained from eight standard solutions, containing $0.7,1.5,3,6$, $12.5,25,50$ and $100 \mu \mathrm{g} / \mathrm{mL}$ of OME in Bis-Tris buffer injected three times. Precision was determined by injecting six times the standard solution containing $12.5 \mu \mathrm{g} / \mathrm{mL}$ of OME. Accuracy was tested using three different standard solutions containing 6, 12.5 and $25 \mu \mathrm{g} / \mathrm{mL}$ of OME injected three times. The repeatability was performed by six standard solutions containing $12.5 \mu \mathrm{g} / \mathrm{mL}$ of OME prepared six times and injected. Specificity was determined by comparing the following solutions: $100 \mu \mathrm{g} / \mathrm{mL}$ of OME alone, in the presence of $\beta C D$ or $M \beta C D$ and in the presence of $\beta C D$ or $M \beta C D$ and ARG injected three times each. At last, the interference of degradation products with OME peak retention time was checked by injection of two different solutions containing $100 \mu \mathrm{g} / \mathrm{mL}$ of OME. In one solution, OME was submitted to degradation in acidic conditions and in the other solution, OME was degraded by high temperatures $\left(60^{\circ} \mathrm{C}\right.$ during $\left.1 \mathrm{~h}\right)$. The final $\mathrm{pH}$ of all solutions was adjusted to $7.0 \pm 0.5$ and relative standard deviations (RSD) were calculated.

\subsection{In vitro permeation studies in porcine buccal mucosa}

\subsubsection{Tissue preparation}

Porcine buccal mucosa was used as it resembles much better the human buccal mucosa regarding permeability, barrier lipid composition, histology and ultrastructural organization [23]. Buccal mucosa from pigs weighing $70-100 \mathrm{~kg}$ was obtained freshly from a local slaughterhouse and it was used at least within $3 \mathrm{~h}$ after animal slaughtering. Most of the underlying tissue was removed from the mucosa with surgical scissors. The buccal tissue 
was dermatomed with a thickness of $500 \mu \mathrm{m}$ [24] using an electrodermatome (Aesculap ${ }^{\circledR}$ Accu Dermatome, Germany).

\subsubsection{Permeation studies}

In vitro permeation studies were conducted in Ussing chambers with a diffusion area of $0.64 \mathrm{~cm}^{2}$ and a compartment volume of $1 \mathrm{~mL}$. All experiments were performed at $37^{\circ} \mathrm{C}$ and protected against light. Dermatomed porcine buccal mucosa was mounted between the donor and the acceptor chambers, which were filled with $40 \mathrm{mM}$ of Bis-Tris buffer at $\mathrm{pH} 7.0 \pm 0.5$ and allowed to equilibrate for $15 \mathrm{~min}$. The donor chamber was emptied after the equilibration period and replaced with $1 \mathrm{~mL}$ of a solution containing $300 \mu \mathrm{g} / \mathrm{mL}$ of OME alone, complexed OME with cyclodextrins $(\beta C D$ and $M \beta C D)$ and complexed OME with cyclodextrins in presence of ARG. Every $30 \mathrm{~min}, 100 \mu \mathrm{L}$ samples were withdrawn from the acceptor chamber and replaced by $100 \mu \mathrm{L}$ of Bis-Tris buffer at $\mathrm{pH} 7.0$ and equilibrated at $37^{\circ} \mathrm{C}$. Samples were analyzed by HPLC in order to determine the amount of permeated OME. Cumulative corrections were made for the previously removed samples.

The apparent permeability coefficients $\left(P_{\text {app }}\right)$ for free and complexed OME were calculated according to the following equation:

$P_{\text {app }}=Q /(A \times c \times t)$, where $Q$ is the total amount permeated within the incubation time $(\mu \mathrm{g}) ; A$ is the diffusion area of the Ussing chamber $\left(\mathrm{cm}^{2}\right) ; c$ is the initial concentration of OME in the donor chamber $\left(\mu \mathrm{g} / \mathrm{cm}^{3}\right)$; $t$ is the total time of the experiment (s).

The cumulative amount of permeated drug was plotted versus time, and the steady state flux $\left(J_{s s}\right)$ was calculated using the formula:

$J_{s s}=\Delta M /(A . \Delta t)$, where $\Delta M$ is the amount of drug transported across the membrane during the time $\Delta t$ and $A$ is the diffusional area.

\subsection{Integrity studies}

FD40 [impermeable fluorescein isothiocyanate (FITC)-labeled dextran] was added to the donor chamber after the permeability measurements (concentration $10 \mathrm{mg} / \mathrm{mL}$ ), and quantified in the receptor chamber, after $1 \mathrm{~h}$ by fluorescence spectroscopy. Concentrations of FD40 were determined using a fluorescence spectrometer Infinite M200 TECAN, at an excitation wavelength of $495 \mathrm{~nm}$ and emission wavelength of $515 \mathrm{~nm}$.

\subsection{Viability studies}

Samples of buccal mucosa of pig were cut, weighed and immersed in Bis-Tris buffer at pH 7.0 0.5 (negative control), in solutions containing inclusion complexes between OME and two cyclodextrins ( $\beta C D$ and $M \beta C D$ ) in the absence and presence of ARG and in a solution with $2 \%(\mathrm{v} / \mathrm{v})$ of Triton, used as positive control. Samples were incubated at $37^{\circ} \mathrm{C}$ and $300 \mathrm{rpm}$ during $3 \mathrm{~h}$. MTT was dissolved $(2 \mathrm{mg} / \mathrm{mL})$ in fresh prepared buffer and filtered through a filter of $0.45 \mu \mathrm{m}$ to remove any dissolved crystals. After $3 \mathrm{~h}, 1 \mathrm{~mL}$ of MTT solution was added to each sample and the samples were placed on a rotating platform (300 rpm) at $37{ }^{\circ} \mathrm{C}$ for $2 \mathrm{~h}$. After this time, the MTT solution was removed and the tissue was rinsed twice with $1 \mathrm{~mL}$ of buffer for $1 \mathrm{~min}$ and then minced with surgical scissors. To extract the water insoluble formazan, $2 \mathrm{~mL}$ of DMSO was added to each sample and stirred (300 rpm) for $80 \mathrm{~min}$ at $37^{\circ} \mathrm{C}$. The absorbance of formazan was measured at $540 \mathrm{~nm}$ (with DMSO as a blank) with the spectrophotometer Infinite M200 TECAN.

\subsection{Cytotoxicity studies}

The same procedure used in viability studies to prepare the samples was carried out. Samples were incubated at $37^{\circ} \mathrm{C}$ while stirred at $300 \mathrm{rpm}$ during $3 \mathrm{~h}$. Each hour a sample was removed and stored at $4{ }^{\circ} \mathrm{C}$. All the samples were centrifuged at $5000 \mathrm{rpm}$ during 2 min to remove possible interferences. Afterwards $50 \mu \mathrm{L}$ of each solution was mixed with $50 \mu \mathrm{L}$ of a specific reagent (Cytotoxicity Detection Kit, Dye solution + Catalyst). Final solutions were incubated at slow stirring for $30 \mathrm{~min}$. The absorbance was measured at $496 \mathrm{~nm}$ with a spectrophotometer Infinite M200 TECAN.

\subsection{Histological studies}

Buccal tissues were cut with the electrical dermatome and incubated at $37{ }^{\circ} \mathrm{C}$ in vials containing Bis-Tris buffer $\mathrm{pH} 7.0 \pm 0.5$, OME solution, complexed OME with cyclodextrins in the absence and presence of ARG and in a solution of Triton $2 \%(\mathrm{v} / \mathrm{v})$. After $3 \mathrm{~h}$, tissue samples were immersed in trypan blue solution during 20 min [25]. After that, samples were washed with buffer and fixed in Bovins solution ( $300 \mathrm{~mL}$ of picric acid, $100 \mathrm{~mL}$ of formaldehyde and $20 \mathrm{~mL}$ of acetic acid). A sample of buccal mucosa, extracted after slaughtering the animal, was used as control and transferred directly to the trypan blue solution and fixed in Bovins solution without any previous incubation. Tissue samples were then washed and dehydrated with a series of isopropanol grades ranging from $70 \%$ to absolute isopropanol, methylbenzoate, chloroform and finally samples were embedded in paraffin. Paraffin preparations were cut into slices and examined under an Olympus BH2 light microscope. The magnification of the microscope used was $10 \times$.

\subsection{Data analysis}

Statistical data analysis was performed using the $t$-test with $p<0.05$ as the minimal level of significance. The statistical analysis was done using GraphPad Prism ${ }^{\circledR}$ version 4.00 software. All experiments were run at least in triplicate.

\section{Results and discussion}

\subsection{Stability studies}

The OME stability in solution was studied in $40 \mathrm{mM}$ of Bis-Tris buffer at different $\mathrm{pH}$ values (Fig. 1). After $3 \mathrm{~h}$ it was observed that $4 \%$ of the OME was degraded at $\mathrm{pH} 7.0$ and 7.9. After $8 \mathrm{~h}$, the remaining drug at $\mathrm{pH} 7.0,7.9$ and 9.0 was $78 \%, 87 \%$ and $100 \%$, respectively. This study confirms that OME degrades at neutral

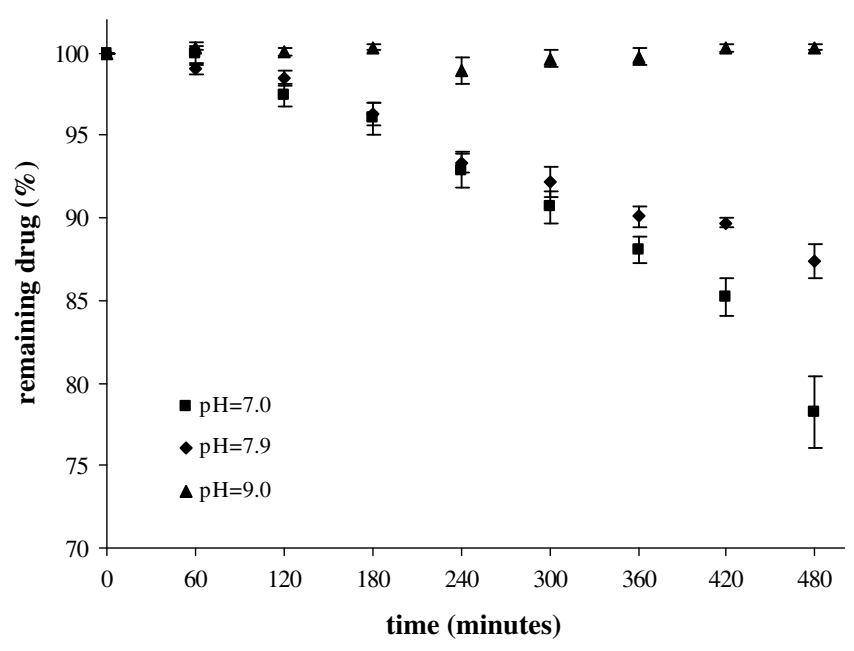

Fig. 1. Stability studies of OME in $40 \mathrm{mM}$ of Bis-Tris buffer at different pH values. Each value is the mean of three independent assays ( $\pm \mathrm{SD}, n=3$ ). 
conditions [26-28]. However, basic conditions are not suitable for drug delivery in physiological membranes. For that reason it is necessary to increase drug stability at neutral conditions in order to develop a pharmaceutical formulation with OME for administration in buccal mucosa.

The stability profiles of OME alone and complexed with $\beta C D$ and $\mathrm{M} \beta C D$ in the absence and presence of ARG are depicted in Fig. 2. Prior to start the stability studies, the $\mathrm{pH}$ of all solutions was adjusted to 7.0 in order to observe the real effect of the inclusion complexation in the absence and presence of ARG in OME stability. After $3 \mathrm{~h}$ the remaining drug calculated was 99\% in the drug complexed with $M \beta C D$ in the presence of ARG and 97\% in the OME complexed with $\beta C D$ in presence of ARG. In the inclusion complex formed in the absence of ARG the remaining drug achieved was $97 \%$ for both cyclodextrins and $96 \%$ in the case of the drug alone. These results show that after $3 \mathrm{~h}$ the complexation with cyclodextrins cannot significantly increase OME stability. However, when the drug was complexed with $M \beta C D$ in the presence of ARG, it was not degraded at all during this period of time. The same result was not obtained for the inclusion complex formed with $\beta C D$ in the presence of ARG. At the end of the study the remaining amount of drug in the solutions containing the drug alone, OME complexed with $\beta C D$ and $M \beta C D$ and the inclusion complexes with ARG, was determined to be $78 \%, 83 \%, 84 \%, 86 \%$ and $91 \%$, respectively. These values indicate that the inclusion of OME in the cyclodextrin cavity increases the OME stability. Nevertheless, in the presence of ARG the improvement on drug stability was more pronounced, especially when the drug was complexed with $M \beta C D$. Previous studies have reported [21] that OME forms a more stable inclusion complex with the $M \beta C D$ than with the $\beta C D$ due to the greater hydrophobic character of the former. Stability studies performed with OME in the presence of ARG at pH 7.0 (data not shown) could not increase OME stability suggesting that ARG alone acts as an alkali agent. These results propose that some interactions can occur between ARG and the inclusion complex. Consequently, ARG can stabilize the inclusion complex formed between OME and cyclodextrins, resulting in increased drug stability at neutral conditions.

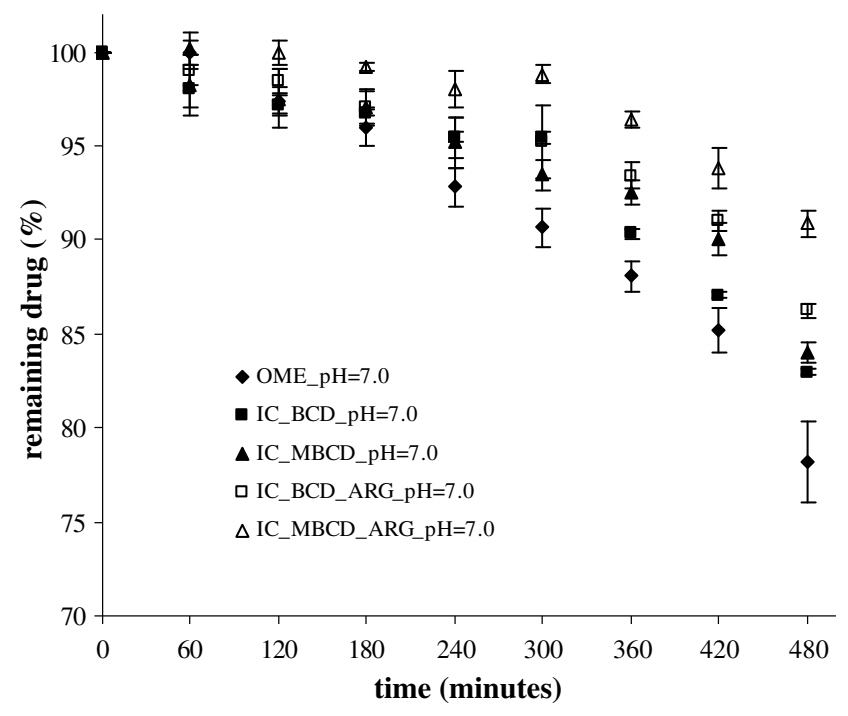

Fig. 2. Stability studies of free and complexed OME with cyclodextrins ( $\beta C D$ and $\mathrm{M} \beta C \mathrm{CD}$ ) in absence and in presence of ARG in $40 \mathrm{mM}$ of Bis-Tris buffer at $\mathrm{pH}$ $7.0 \pm 0.5$. Each value is the mean of three independent assays $( \pm \mathrm{SD}, n=3)$. Inclusion complex between OME and $\beta C D$ (IC_BCD); inclusion complex between OME and $M \beta C D$ (IC_M $\beta C D$ ); inclusion complex between OME and $\beta C D$ in presence of ARG (IC_BCD_ARG) and inclusion complex between OME and $\mathrm{M} \beta C D$ in presence of ARG (IC_MBCD_ARG).

\subsection{Validation of the HPLC method}

The calibration curve and regression coefficient for the proposed method were: $Y=203742 X-140888$ and $R^{2}=0.9998$ indicating a good linearity in the range of the study. The $R^{2}$ obtained was higher than 0.999, as frequently recommended [29]. RSD values calculated are showed in Table 1 . The results show a good precision, accuracy, repeatability and specificity of the analytical method. At last, results confirm that the presence of cyclodextrins $(\beta C D$ and $M \beta C D$ ), alkali agent (ARG) or degradation products do not interfere with the retention time corresponding to OME peak.

\subsection{Permeation studies}

In vitro permeability studies are an useful tool to assess the potential of a localized anatomical site as a route for drug delivery. However, in vitro conditions should simulate the in vivo situation as closest possible.

The permeability profiles of OME alone, complexed with $\beta C D$ and $\mathrm{M} \beta C D$ and complexed OME in presence of $A R G$, are shown in Fig. 3A and B. Table 2 contains apparent permeability coefficients $\left(P_{\mathrm{app}}\right)$ and flux values of the different studied systems. The cumulative amount of complexed OME with $\beta C D$ absorbed over $3 \mathrm{~h}$ through epithelium was only 1.1 -fold greater than the amount of absorbed OME alone. In OME complexed with M $\beta C D$ the increase in drug absorbed was 1.4-fold compared to the OME alone. These results suggest that $\beta C D$ acts as a carrier, keeping the hydrophobic OME molecules in solution and delivering them to the surface of the buccal mucosa. In the case of $M \beta C D$, the enhancement in drug absorption indicates that this cyclodextrin acts as an enhancer of permeation by different mechanisms as reported in the literature $[11,12]$. Lipophilic cyclodextrins such as $M \beta C D$, can permeate biomembranes, interact with the lipids and increase drug uptake through the buccal mucosa. Permeation studies with OME complexed with both cyclodextrins ( $\beta C D$ and $M \beta C D$ ) in the presence of ARG show an enhancement of 1.7- and 2.4-fold, respectively, when compared with the amount of absorbed OME alone. This fact suggests that beyond the permeation enhancement effect of the cyclodextrins, the presence of ARG can stabilize the inclusion complex formed and, this stabilizing effect of the OME at the biomembrane surface increases the amount of OME available to permeate the buccal epithelium. On the other hand, a recent study [30] shows that the poly-L-arginine can act as an enhancer of the mucosal epithelia permeability by positively charged amino groups, interacting with negatively charged sites on the epithelial membrane and tight junctions. At neutral conditions, ARG is in the cationic form being able to interact with negatively charged molecules situated in the mucus layer [31] on the surface of the buccal

Table 1

RSD values of the analytical parameters used to validate the HPLC method

\begin{tabular}{lc}
\hline Standard solution $(\mu \mathrm{g} / \mathrm{mL})$ & RSD $\%$ \\
\hline Precision $(n=6)$ & 2.207 \\
12.5 & \\
Accuracy $(n=9)$ & 2.515 \\
6 & 0.715 \\
12.5 & 0.599 \\
25 & \\
Repeatibility $(n=6)$ & 3.454 \\
12.5 & \\
Specificity $(n=9)$ & 3.314 \\
100 & 0.721 \\
$100+\beta C D$ & 0.247 \\
$100+$ M $\beta C D$ & 0.308 \\
$100+\beta C D+$ ARG & 0.979 \\
$100+$ M $\beta C D+$ ARG & \\
\hline
\end{tabular}



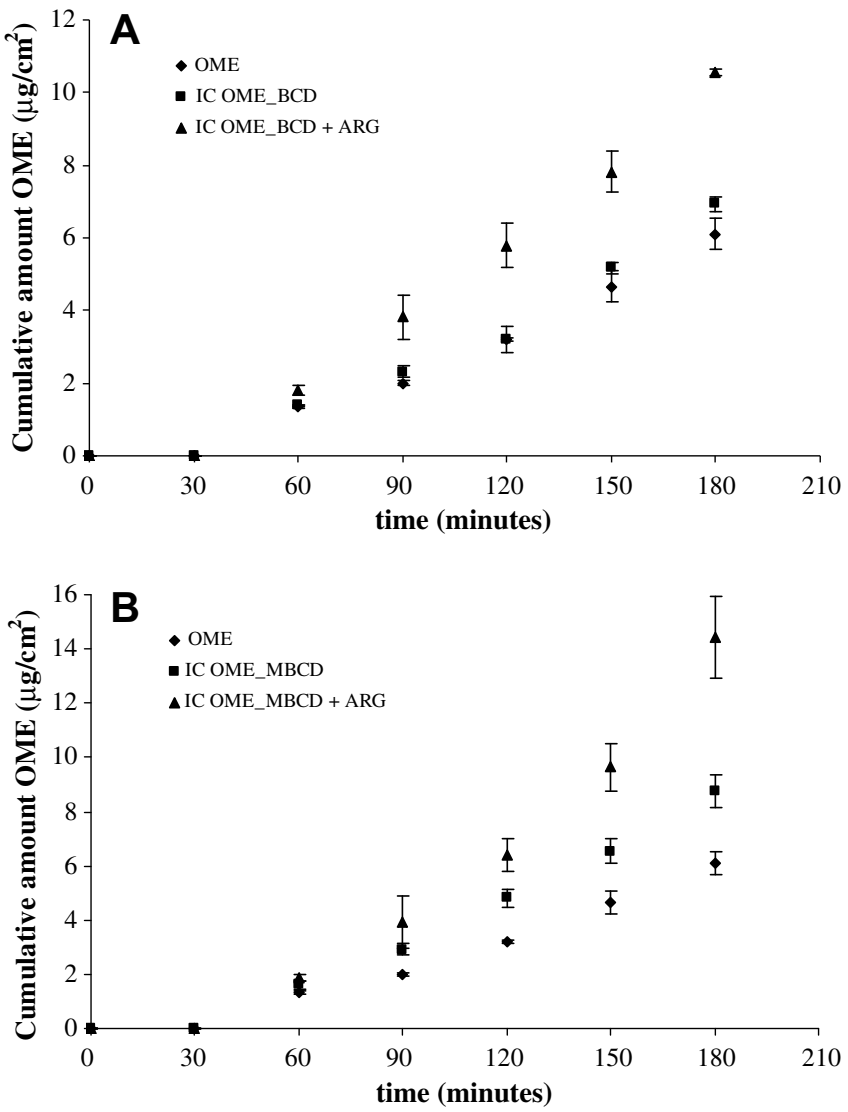

Fig. 3. In vitro permeation studies in porcine buccal mucosa of free and complexed OME with $\beta C D(A)$ and $M \beta C D(B)$ in the absence and in presence of ARG solution. Each value is the mean of three assays $( \pm \mathrm{SD}, n=3)$.

Table 2

Apparent permeability coefficients $\left(P_{\mathrm{app}}\right)$ and flux values of different studied systems

\begin{tabular}{|c|c|c|c|}
\hline Systems & $P_{\text {app }}(\mathrm{cm} / \mathrm{s})$ & Ratio $^{a}$ & Flux $\left(\mu \mathrm{g} / \mathrm{cm}^{2} \mathrm{~h}\right)$ \\
\hline OME & $1.883 \mathrm{E}-06 \pm 7.692 \mathrm{E}-08$ & 1.0 & $2.382 \pm 0.185$ \\
\hline IC OME_ $\beta C D$ & $2.143 \mathrm{E}-06 \pm 3.638 \mathrm{E}-08$ & 1.1 & $2.685 \pm 0.092$ \\
\hline IC OME_M $\beta C D$ & $2.702 \mathrm{E}-06 \pm 1.040 \mathrm{E}-07$ & 1.4 & $3.455 \pm 0.217$ \\
\hline IC OME_ $\beta C D+$ Arg & $3.269 \mathrm{E}-06 \pm 1.900 \mathrm{E}-08$ & 1.7 & $4.161 \pm 0.117$ \\
\hline IC OME_M $\beta C D+$ Arg & $4.445 \mathrm{E}-06 \pm 2.686 \mathrm{E}-07$ & 2.4 & $5.588 \pm 0.564$ \\
\hline
\end{tabular}

Indicated values are means $( \pm \mathrm{SD}, n=3-4)$.

${ }^{\text {a }}$ Enhancement ratio $=P_{\text {app }}($ sample $) / P_{\text {app }}($ control $)$.

mucosa. This suggestion is supported by the permeation studies performed with OME in presence of ARG (data not shown) was observed a 1.2-fold increase in the amount of permeated drug in presence of ARG compared to the amount of OME alone permeated.

In the integrity studies, fluorescence values calculated for all the systems after $1 \mathrm{~h}$ of incubation with a solution containing $10 \mathrm{mg} /$ $\mathrm{mL}$ of FD40, indicated a concentration of FD40 in the acceptor chamber below $0.01 \%$. This data suggests that the tissue integrity of buccal mucosa is maintained during the permeation studies. The principle here is that FD40 being a very large molecule, cannot permeate the buccal mucosa and enter the acceptor chamber unless the mucosa is damaged.

\subsection{Viability studies}

The MTT assay has been used for quantitative colorimetric measurements of mammalian cell survival and proliferation. The original assay has been modified to assess the viability of the tissue specimens [32].
MTT is converted in viable cells to formazan (a dark purple water insoluble compound) by enzymes in active mitochondria collectively known as tetrazolium reductase and the amount of formazan generated is directly proportional to the number of living cells [33]. The results of cell viability (\%) after $3 \mathrm{~h}$ of incubation of porcine buccal mucosa with samples solutions, negative and positive control are shown in Fig. 4. To calculate the percentage of cell viability, sample in Bis-Tris buffer (negative control) was used as $100 \%$ of cellular viability. It was observed that cell viability of the buccal mucosa after $3 \mathrm{~h}$ of incubation remained around $70 \%$ in all samples as compared to Bis-Tris buffer (negative control). By contrast, Triton $2 \%(\mathrm{v} / \mathrm{v})$ treated cells displayed a significant decrease $(p<0.05)$ of viability after $3 \mathrm{~h}$ of incubation (around 30\%) when compared with negative control and all sample solutions.

\subsection{Cytotoxicity studies}

Cell death is typically assayed by quantifying plasma membrane damage and in recent years, lactate dehydrogenase (LDH) activity has been used in toxicological studies in mucosal membranes $[34,35]$. LDH is a stable cytoplasmic enzyme which is present in all cells. When the plasmatic membrane is damaged, LDH is rapidly released into the supernatant medium and its activity can be determined by a colorimetric reaction. In the first step $\mathrm{NAD}^{+}$is reduced to $\mathrm{NADH} / \mathrm{H}^{+}$by the $\mathrm{LDH}$-catalyzed conversion of lactate to pyruvate. In the second step, a catalyst added to the supernatant medium (diaphorase) transfers $\mathrm{H} / \mathrm{H}^{+}$from $\mathrm{NADH} / \mathrm{H}^{+}$to the tetrazolium salt, which was reduced to a formazan dye. An increase in the number of dead or plasma membrane-damaged cells leads to an increased LDH activity in the supernatant medium, which directly correlates with the amount of formazan produced. Therefore, the amount of dye produced is proportional to the number of lysed (dead or plasmatic membrane damaged) cells [36].

Methylated $\beta$-cyclodextrins interact strongly with lipids [11], for that reason, cell membrane integrity was evaluated by quantification of the LDH released. Until 1 and 3 h, no significant differences in LDH activity (absorbance of formazan/mg of tissue) were observed in the samples incubated with inclusion complexes between OME and both cyclodextrins ( $\beta C D$ and $M \beta C D$ ) in absence

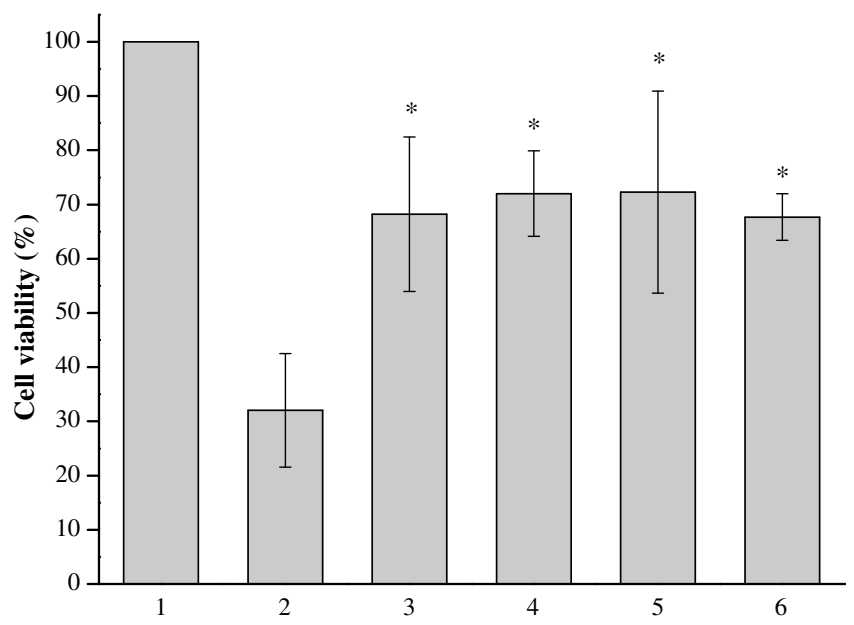

Fig. 4. Cell viability in porcine buccal mucosa measured by MTT assay expressed as the mean values obtained from three inserts $( \pm S D, n=3)$. (1) Buccal mucosa after $3 \mathrm{~h}$ exposed to Bis-Tris buffer $40 \mathrm{mM}$ (positive control); (2) buccal mucosa after $3 \mathrm{~h}$ exposed to Triton $2 \%(\mathrm{v} / \mathrm{v}$; negative control); ( 3 ) buccal mucosa after $3 \mathrm{~h}$ exposed to OME_ $\beta C D$ inclusion complex in solution; (4) buccal mucosa after $3 \mathrm{~h}$ exposed to OME_M $\beta C D$ inclusion complex in solution; (5) buccal mucosa after $3 \mathrm{~h}$ exposed to OME_ $\beta C D$ inclusion complex in ARG solution; (6) buccal mucosa after $3 \mathrm{~h}$ exposed to OME_M $\beta C D$ inclusion complex in ARG solution; "all these samples are statistically different compared to the sample exposed to Triton $2 \%(\mathrm{v} / \mathrm{v}),(p<0.05)$. 


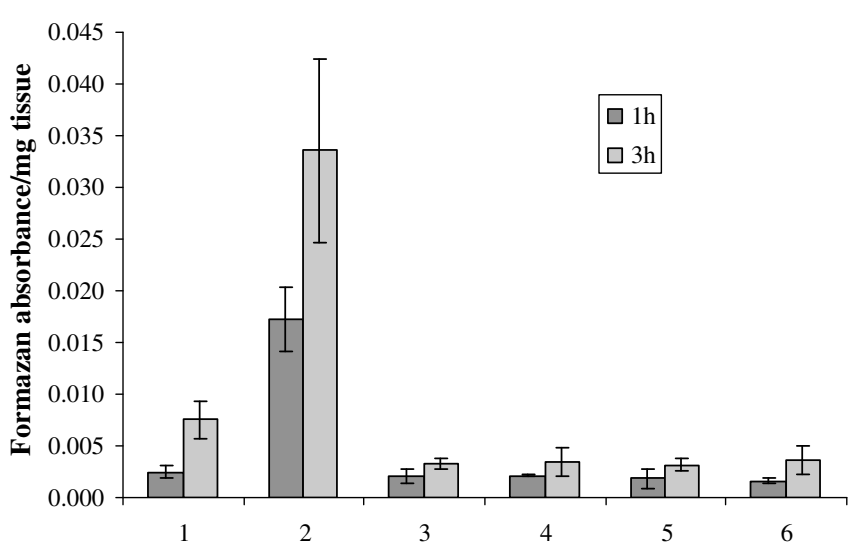

Fig. 5. Formazan absorbance resultant of LDH activity released from porcine buccal mucosa after 1 and $3 \mathrm{~h}$ exposed to: (1) Bis-Tris buffer $40 \mathrm{mM}$; (2) Triton 2\% (v/v) solution; (3) OME_ $\beta C D$ inclusion complex in solution; (4) OME_M $\beta C D$ inclusion complex in solution; (5) OME_ $\beta C D$ inclusion complex in ARG solution; (6) OME_M $\beta C D$ inclusion complex in ARG solution.

and in presence of ARG and the sample exposed to Bis-Tris buffer $40 \mathrm{mM}$ (Fig. 5). At the same time, the sample exposed to Triton $2 \%$ $(\mathrm{v} / \mathrm{v})$ showed a significant increase in the absorbance of formazan between 1 and $3 \mathrm{~h}(p<0.05)$. These results demonstrate that cyclodextrins, namely $\beta C D$ and $M \beta C D$ even in presence of an alkali agent, ARG, do not present cytotoxic effects to the porcine buccal mucosa when compared with Triton solution.

\subsection{Histological studies}

Fig. 6 shows the photomicrographs of the buccal epithelium after slaughter the animal, $3 \mathrm{~h}$ after incubation at $37^{\circ} \mathrm{C}$ with BisTris buffer $\mathrm{pH} 7.0 \pm 0.5$, OME alone, complexed with cyclodextrins in the absence or presence of ARG and in a solution of Triton $2 \%$ $(\mathrm{v} / \mathrm{v})$, which is known to be extensively toxic [37]. In some photomicrographs it was possible to observe a separation of the epithelium from the connective tissue and only the buccal epithelium is present [38]. Histological studies showed that after $3 \mathrm{~h}$ of incubation with sample solutions there were no cell leakage and no visible changes in the thickness of the superficial layer was observed, indicating that the buccal epithelium appeared viable when compared with the tissue incubated with buffer or the tissue removed immediately after death of the animal. Only few cells in the superficial layer of some preparations appeared to be dead, probably due to the mechanical stress during tissue preparation. However, the thickness of the buccal epithelium was greatly reduced after $3 \mathrm{~h}$ exposed to a Triton solution. Treatment of buccal epithelium with this solution resulted in cellular changes and tissue necrosis probably due the detergent effect of this compound. These histological results support data obtained in the viability and cytotoxicity studies.

\section{Conclusion}

Buccal drug delivery offers an alternative to conventional oral administration for drugs that show low stability at acidic conditions of the stomach and a strong first hepatic effect. However, buccal mucosa represents an effective absorption barrier and new strategies must be found to overcome it. The effect of cyclodextrins to increase OME stability and permeability through the buccal epithelium was studied and the integrity, viability and cytotoxicity effects were evaluated after the exposition of the buccal tissue with this kind of permeation enhancers. Stability studies performed at neutral conditions suggested that the complexation of OME increased drug stability and in the presence of ARG this effect was improved. The in vitro transbuccal permeation of OME was enhanced in the presence of cyclodextrins. This increase was highest with $\mathrm{M} \beta C D$ indicating that this lipophilic cyclodextrin can permeate buccal mucosa and consequently to enhance drug delivery through the biomembrane. The complexation of OME with $\mathrm{M} \beta C D$ in presence of ARG increases drug permeation 2.4-fold, suggesting that ARG favors drug permeation due to the establishment of ionic interactions with negative charges present in mucus layer on the surface of the mucosa. Viability studies showed that buccal mucosa remains viable after $3 \mathrm{~h}$ incubation period with OME in complexed form in absence and in presence of ARG. These results were supported by histological evaluations. No toxicity effects
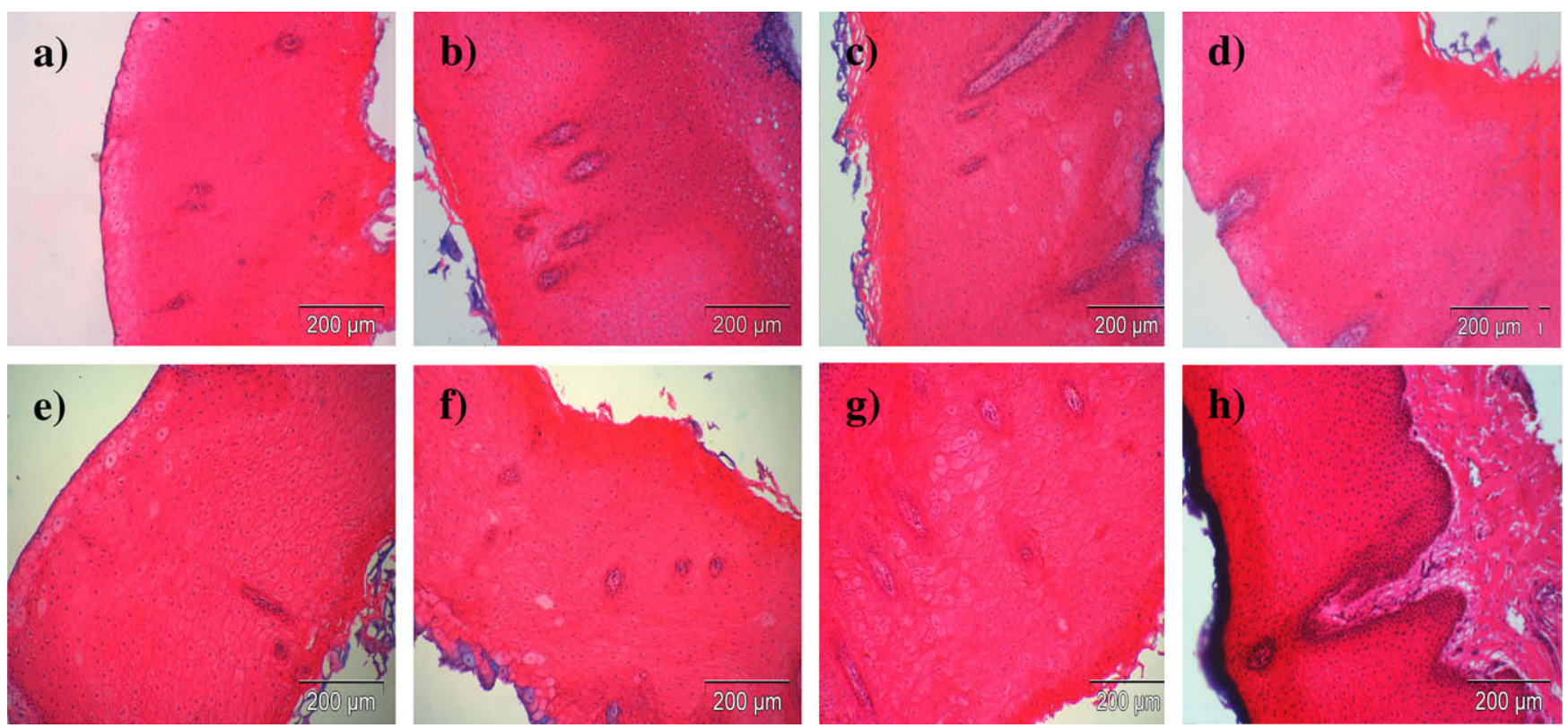

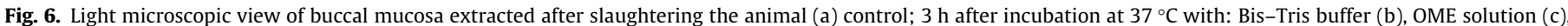

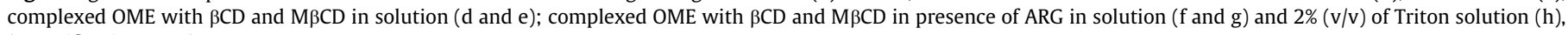
(magnification, $10 \times$ ). 
were observed in buccal mucosa after incubation with OME complexed with cyclodextrins even in the presence of ARG. The results presented here demonstrate that complexation of OME with $M \beta C D$ in presence of ARG may be promising approach to increase drug stability and permeation through the buccal mucosa, that ultimately can result in improve drug bioavailability.

\section{Acknowledgements}

This work was financially supported by a grant (Praxis SFRH/ BD/19175/2004) from FCT (Fundação para a Ciência e a Tecnologia, Portugal). The authors thank the technical assistance of Prof. Klima and Rudolf Haring (Department of Histology and Embryology, University of Innsbruck) in the preparation and visualization of the histological samples and Dr. Christian Lei $\beta$ (Department of Pharmaceutical Technology, Faculty of Pharmacy, University of Innsbruck) in the preparation of buccal tissue for the permeation studies. We also acknowledge Belmac Laboratory, S.A. (Madrid, Spain) for the kindly donating the OME and Roquette (Lestrem, France) for the $\beta C D$ and $M \beta C D$ samples.

\section{References}

[1] M.E. De Vries, H.E. Bodde, J.C. Verhoef, H.E. Junginger, Developments in buccal drug delivery, Crit. Dev. Ther. Drug Carrier Syst. 8 (1991) 271-303.

[2] I.D. Del Consuelo, G. Pizzolato, F. Falson, R.H. Guy, Y. Jacques, Evaluations of pig esophageal mucosa as a permeability barrier model for buccal tissue, J. Pharm. Sci. 94 (2005) 2777-2788.

[3] Y. Sudhakar, K. Kuotsu, A.K. Bandyopadhyay, Buccal bioadhesive drug delivery - a promising option for orally less efficient drugs, J. Control. Release 114 (2006) 15-40

[4] S. Senel, A.A. Hincal, Drug permeation enhancement via buccal route: possibilities and limitations, J. Control. Release 72 (2001) 133-144.

[5] T. Loftsson, M.E. Brewster, M. Másson, Role of cyclodextrins in improving oral drug delivery, Am. J. Drug Deliv. 2 (2004) 1-15.

[6] L. Matilainen, T. Toropainen, H. Vihola, J. Hirvonen, T. Järvinen, P. Jarho, K Järvinen, In vitro toxicity and permeation of cyclodextrins in Calu-3 cells, J. Control. Release 126 (2008) 10-16.

[7] K.H. Fromming, J. Szejtli, Cyclodextrin in Pharmacy, Kluwer Academic Publishers, Dordrecht, 1994

[8] V.J. Stella, R.A. Rajewski, Cyclodextrins: their future in drug formulation and delivery, Pharm. Res. 14 (1997) 556-567.

[9] T. Loftsson, M.E. Brewster, Pharmaceutical applications of cyclodextrins. I: Drug solubilization and stabilization, J. Pharm. Sci. 85 (1996) 1017-1025.

[10] T. Loftsson, N. Bodor, Percutaneous Penetration Enhancers, CRC Press, Boca Raton, 1995.

[11] T. Irie, K. Wakamatsu, H. Arima, H. Aritomi, K. Uekama, Enhancing effects of cyclodextrins on nasal absorption of insulin in rats, Int. J. Pharm. 84 (1992) 129-139.

[12] M. Masson, T. Loftsson, G. Másson, E. Stefánsson, Cyclodextrins as permeation enhancers: some theoretical evaluations and in vitro testing, J. Control. Release 59 (1999) 107-118.

[13] C.A. Squier, P.S. Cox, P.W. Wertz, D.T. Downing, The lipid composition of porcine epidermis and oral epithelium, Arch. Oral Biol. 31 (1986) 741-747.

[14] P.W. Wertz, D.C. Swartzendruber, C.A. Squier, Regional variation in the structure and permeability of oral mucosa and skin, Adv. Drug Deliv. Rev. 12 (1993) 1-12.

[15] C.A. Squier, P.W. Wertz, Permeability and the pathophysiology of oral mucosa, Adv. Drug Deliv. Rev. 12 (1993) 13-24.

[16] G.L. Kearns, T. Andersson, L.P. James, A. Gaedigk, R.A. Kraynak, S.M. AbdelRahman, K. Ramabadran, J.N. van den Anker, Omeprazole disposition in children following single-dose administration, J. Clin. Pharmacol. 43 (2003) $840-848$

[17] D. Castro, M.A. Moreno, S. Torrado, J.L. Lastres, Comparison of derivative spectrophotometric and liquid chromatographic methods for the determination of omeprazole in aqueous solutions during stability studies, J. Pharm. Biomed. Anal. 21 (1999) 291-298.

[18] T. Andersson, R. Bergstrand, C. Cederberg, Influence of acid secretory status on absorption of omeprazole from enteric coated granules, Br. J. Clin. Pharmacol. 31 (1991) 275-278.

[19] A.B. Thomsom, P. Kirdeikis, R. Lastiwka, K. Rohss, P. Sinclair, B. Olofsson, Pharmacokinetics and pharmacodynamics during treatment with the omeprazole $20 \mathrm{mg}$ enteric coated tablet and $20 \mathrm{mg}$ capsule in asymptomatic duodenal ulcer patients, Can. J. Gastroenterol. 11 (1991) 657-660.

[20] A. Figueiras, R.A. Carvalho, L. Ribeiro, J.J. Torres-Labandeira, F.J.B. Veiga, Solidstate characterization and dissolution profiles of the inclusion complexes of omeprazole with native and chemically modified $\beta$-cyclodextrin, Eur. J. Pharm. Biopharm. 67 (2007) 531-539.

[21] A. Figueiras, J.M.G. Sarraguça, R.A. Carvalho, A.A.C.C. Pais, F.J.B. Veiga, Interaction of omeprazole with a methylated derivative of $\beta$-cyclodextrin: phase solubility, NMR spectroscopy and molecular simulation, Pharm. Res. 24 (2007) 377-389.

[22] USP XXX. The United States Pharmacopoeia, 25th ed., United States Pharmacopeial Convention, Inc., Rockville, 2007.

[23] N. Langoth, A. Bernkop-Schnürch, P. Kurka, In vitro evaluation of various buccal permeation enhancing systems for PACAP (pituitary adenylate cycloseactivating polypeptide), Pharm. Res. 22 (2005) 2045-2050.

[24] A.H. Shojaei, Buccal mucosa as a route for systemic drug delivery: a review, J. Pharm. Pharm. Sci. 1 (1998) 15-30.

[25] N. Langoth, J. Kalbe, A. Bernkop-Schnürch, Development of a mucoadhesive and permeation enhancing buccal delivery system for PACAP (pituitary adenylate cyclase-activating polypeptide), Int. J. Pharm. 296 (2005) 103111.

[26] M. Mathew, V.D. Gupta, R.E. Baile, Stability of omeprazole solution at various $\mathrm{pH}$ values as determined by high performance liquid chromatography, Drug Dev. Ind. Pharm. 21 (1995) 965-971.

[27] N. Sarisuta, T. Tourtip, S. Chuarcharoern, Chemical stability and mechanism of degradation of omeprazole in solution, Thai. J. Pharm. Sci. 22 (1998) 81-88.

[28] A. Riedel, C.S. Leopold, Degradation of omeprazole induced by enteric polymeric solutions and aqueous dispersion HPLC investigations, Drug Dev. Ind. Pharm. 31 (2005) 151-160.

[29] N. Épshtein, Validation of HPLC techniques for pharmaceutical analysis, Pharm. Chem. J. 38 (2004) 212-228.

[30] G. Di Colo, Y. Zambito, C. Zaino, Polymeric enhancers of mucosal epithelia permeability: synthesis, transepithelial penetration-enhancing properties, mechanism of action, safety issues. J. Pharm. Sci. (2007), published online in Wiley InterScience, doi: 10.1002/jps.21043.

[31] A. Mikos, N. Peppas, Systems for controlled release of drugs. V: Bioadhesive systems, STP Pharma 2 (1986) 705-716.

[32] D. Imbert, C. Cullander, Buccal mucosa in vitro experiments. I: Confocal imaging of vital staining and MTT assays for the determination of tissue viability, J. Control. Release 58 (1999) 39-50.

[33] J.A. Nicolazzo, B.L. Reed, B.C. Finnin, The effect of various in vitro conditions on the permeability characteristics of the buccal mucosa, J. Pharm. Sci. 92 (2003) 2399-2410.

[34] C. Callens, E. Adriaens, K. Dierckens, S.P. Remon, Toxicological evaluation of a bioadhesive nasal powder containing a starch and carbopol 974P on rabbit nasal mucosa and slug mucosa, J. Control. Release 76 (2001) 81-91.

[35] P. Sharma, M.V. Varma, H.P. Chawla, R. Panchagnula, In situ and in vivo efficacy of peroral absorbtion enhancers in rats and correlation to in vitro mechanistic studies, IL Fármaco 60 (2005) 874-883.

[36] J.A. Cook, J.B. Muchell, Viability measurements in mammalian cell systems, Anal. Biochem. 179 (1989) 1-7.

[37] E.M. Zimmerman, Z. Vaituzis, F.M. Hetrick, Mitochondrial damage and inhibition of respiration in animal cell cultures treated with Triton WR1339, J. Cell. Physiol. 74 (2005) 67-76.

[38] A.J. Hoogstraate, H.E. Bodde, Methods for assessing the buccal mucosa as a route of drug delivery, Adv. Drug Deliv. Rev. 12 (1993) 99-125. 